\title{
DÉCLENCHEMENT DE L'CESTRUS ET OBTENTION DE LA GESTATION PENDANT L'ANCESTRUS POST-PARTUM CHEZ LES BREBIS A L'AIDE D'ÉPONGES VAGINALES IMPRÉGNÉES D'ACÉTATE DE FLUOROGESTONE (')
}

\author{
J. THIMONIER, P. MAULÉON, Y. COGNIÉ, R. OR'TAVANT \\ avec la collaboration technique de C. Cornu et C. Matre \\ Laboratoire de Physiologie de la Reproduction, \\ Centre de Recherches vétérinaires et zootechniques, 37 - Nouzilly \\ Institut national de la Recherche agronomique
}

\section{SOMMAIRE}

Les facteurs qui agissent sur le déclenchement hormonal de l'œstrus et sur l'établissement de la gestation pendant l'ancstrus post-parium chez les ovins ont été étudiés à différentes périodes de l'année.

L'œstrus peut être induit avec un traitement progestatif par voie vaginale, 32 à 35 jours après la parturition, quelie que soit la période de l'année, chez 80 à $90 \mathrm{p}$. Ioo des brebis. Une supplémentation PMSG est cependant indispensable pour obtenir un pourcentage élevé de brebis en œstrus pendant la période d'anoestrus.

La fertilité après l'œstrus induit est faible mais elle augmente lorsque l'intervalle mise basdébut du traitement croît. Chez les brebis de race Ile-de-France, le pourcentage de fertilité passe de Io à 35 , lorsque l'intervalle parturition-début du traitement progestatif varie de 17 à 25 jours.

Il existe des différences raciales. La gestation lors de l'cestrus induit s'établit plus facilement chez les brebis de race Préalpes que chez les brebis de race Ile-de-France. Pour un même intervalle après mise bas, en période d'anœestrus, les pourcentages de fertilité après l'œestrus induit, sont respectivement de 32 et 55 , chez les brebis Ile-de-France et Préalpes.

Enfin, les brebis taries présentent un pourcentage de retours en ostrus chez les animaux non gestants plus élevé que les brebis allaitantes. Il est possible ainsi d'améliorer la fertilité globale, particulièrement en période d'anœestrus.

\section{INTRODUC'TION}

Deux causes principales empêchent de réduire l'intervalle entre deux gestations chez les ovins : l'existence d'un anœstrus saisonnier au printemps et l'apparition d'un ancestrus post-partum, pendant la saison sexuelle. La durée de l'ancestrus post-partım dépend de la race considérée. Elle varie suivant que les brebis sont allaitantes

(1) Acétate d a fluorogestone $=$ F. G. A. $=$ I $7 \alpha$ acétoxy $9 \alpha$ fluoro i $\beta$ hydroxyprogestérone $=$ bC 9880 . Produit Searle. 
ou taries. Chez des brebis Rambouillet allaitantes, la durée de cet anostrus est de 4I jours en moyenne (Granger, I947). Elle n'est que de 24 jours, chez des brebis Rambouillet $\times$ Down taries (WILliams et al., I956). Pour les animatix de la race Ile-de-France, sa durée est de 59 jours, mais le nombre de brebis qui viennent en chaleurs avant cette période est plus grand pour les brebis taries, que pour celles qui allaitent leurs agneaux (Mauléor et Dauzier, I965). Après cette courte période d'anœstrus, il est possible de faire reproduire les animaux avec un taux élevé de fertilité (GRANGER, I947 ; HAFEZ, I952).

En période d'ancestrus saisonnier, l'intervalle mise bas-premier cestrus se confond, pour toutes les races, avez l'intervalle mise bas-début de 1a saison de reproduction (LAEES, I964; Mauléon et DauzIer, I965). Deux injections de PMSG à I6 jours d'intervalle (COLE et MILLER, I933; ZAVADOVSKY, I945) ou un traitement associant progestérone et PMSG (DAUZIER et WINTENBERGER, I952; DUTT, I952 ; ROBINSON, I954; GORDON, I958-I963a et b) permettent d'induire l'cstrus et d'obtenir la gestation chez 30 à 70 p. Ioo des animaux pendant l'anœestrus saisonnier. Si l'ancestrus de lactation interfère avec l'anœstrus saisonnier, l'induction de l'ostrus est plus difficile et la fertilité est alors très faible: taux de conception de I4 p. IOo (GoRdon, I958) et de o p. IoO (ALLEN et LAMming, I960).

L'utilisation des stéroïdes de synthèse a permis d'augmenter le pourcentage de brebis venant en œstrus, mais le problème de la fertilité n'a pas été résolu. Les traitements ne doivent pas débuter moins de 2 mois après la parturition si l'on veut obtenir une fertilité de $60 \mathrm{p}$. Ioo que les brebis soient allaitantes ou non, (HANSEL, I964; WAGNer, I964; HulET et Foote, I967 $a$; RAHMAN et KitTs, I967).

Or l'obtention de deux agnelages par an suppose l'établissement de la gestation 3 o à 35 jours après mise bas (Mauréon, Pinot et du Messir, du Buisson, ig65). L'état de l'utérus peu de temps après la mise bas peut expliquer la faible fertilité observée chez les brebis allaitantes. HEAP, ALLEN et LAMMING (I963) ont montré le rôle important des ovulations silencieuses qui se produisent après la mise bas avant la reprise du comportement sexuel cyclique sur l'amélioration du milieu utérin.

La fertilité est augmentée si l'accouplement est effectué lors d'un œstrus précédé par un cycle ovarien (Gordon, I958).

Le but de cette étude a donc été de découvrir les facteurs qui permettent d'ac. croître la venue en ostrus et la fertilité des brebis peu de temps après la mise bas, à différentes périodes de l'année.

\section{MATÉRIEL E'T MÉTHODES}

\section{I ${ }^{\circ}$ Matériel animal}

Les différentes expérimentations ont été effectuées sur des animaux de races Ile-de-Franct t Préalpes.

L.e troupeau Préalpes est celui du laboratoire (latitude $47^{\circ} 30^{\prime}$ Nord). Les aninaux vivent uniquement en bergerie. Les brebis de cette race, grâce à un anostrus saisonnier court peuvent donner 3 agnelages en 2 ans, dans leur région d'origine (latitude $45^{\circ} \mathrm{N}$ ).

Les expérimentations sur la race Ile-de-France ont été effectuées sur le troupeau du laboratoire dont la saison sexuelle se situe normalement à l'automne. Elles ont aussi été réalisées dans les élevages du Bassin Parisien, (latitude $49^{\circ} 30^{\prime} \mathrm{N}$ ) qui pratiquent avec un certain succès une lutte de 
printemps au mois de mai. Daus ce cas, par une conduite d élevage appropriée, les éleveurs st imulent la reprise transitoire d'activité ovarienne normalement observée en mai (Tinmoxier et MaLLÉoN. I 967$)$.

\section{Traitements}

Nous avons adopté pour nos expérimentation, le même progestagène (FG.A) et le méne mode d'administration (éponges vaginales) que ceux utilisés par RobINSON (I965). Quatre doses d'acétate de fluorogestone : $20,30,45$ et $60 \mathrm{mg}$ ont été déposées sur des éponger en polyuréthane.

Des doses différentes d'normone gonadotrope sérique PMSG:0, 400, 600, 800, i 200 UI ont été injectées soit 2 jours avant, soit immédiatement, soit i 2 heures après le retrait des éponges.

0,25 ou $50 \mu \mathrm{g}$ de benzoate ou de valérianate d'cestradiol ont été injectés par voie sous-cutanée ¿ la fin du traitement progestatif. Le benzoate d'ocstradiol a été quelquefois administré au début observé des chaleurs induites.

Le traitement avec le progestagène a débuté soit $17 \pm 2$ jours, soit $25 \pm 3$ jours après la mise bas et a duré 12, 14, ou 17 jours selon les expérimentations. Les saillies ont eu lieu soit $35 \pm 5$ jours, soit $43 \pm 6$ jours aprés la parturition.

L'influence de l'allaitement (brebis taries 2 a 4 jours après la mise bas ou brebis allaitantes) a été étudiée. Pour contrôler le rôle d'une ovulation silencieuse sur le milieu utérin, 800 UI de PMSG ont été injectées $\mathbf{2}$ jours avant le début du traitement avec le progestagìne.

Quelles que soient les expérimentations, les brebis témoins étaient allaitantes ; il n'a pas été fait de lot de brebis témoins taries. Les brebis ayant perdu leur éponge ont été éliminées des calculs.

\section{$3^{\circ}$ Détection de l'ostrus et saillie}

La détection de l'oestrus a été faite deux fois par jour, pendant les 5 jours suivant la fin du traitement puis une fois par jour pendant vingt jours, selon la technique précisée précédemment par l'un de nous (MAULÉon et DAL"ZIER, I 965).

1)ans les cas où des erreurs de détection de l'cestrus ont été observées, la date de saillie a pu être déterminée en tenant compte de la date de la mise bas.

Les brebis ont été saillies naturellement. Dans un seul cas elles ont été inséminées artificielle. ment ( 9 I brebis Ile-de-France du laboratoire, printemps i967).

\section{$4^{\circ}$ Définitions}

Nous avons modifié les détinitions données par Desvicines et DARPoux (1964) pour les adapter à nos conditions.

\section{a) Brebis traile'es.}

Nous appelons pourcentage de brebis en astrus, le nombre moyen de brebis entrant en chaleurs moins de 5 jours après le retrait de l'épongs vaginale, pour oo brebis traitées.

Le pourcentage de retours en cestrus est le nombre moyen de brebis revenant en cestrus après une saillie non fécondante, pour roo brebis non gestantes après cette première saillie.

Celui de fertilité est le nombre moyen de brebis mettant bas pour 100 brebis traitées.

Nous avons calculé le pourcentage de mise bas après l'ostrus induit par le traitement progestatif, celui de la fertilité après 5 jours de lut te (chaleurs induites) et celui de fertilité globale calculé pour toute la durée de l'expérimentation ( 2 cestrus consécutifs après la fin du traitement progestatif).

\section{b) Brebis témoins.}

Le pourcentage de brebis en ostrus a été calculé pour 2 périodes différentes après la parturition : moins de 42 jours et moins de 5 I jours. Un intervalle de temps pour l'apparition du premier astrus identique à celui des brebis traitées $17 \pm 2$ jours et $25 \pm 3$ jours, après la mise bas, est ainsi alloué aux brebis témoins.

Le pourcentage de mise bas a été établi pour ces 2 périodes ainsi que pour un intervalle moyen parturition-fin de la lutte, identique à celui des animaux traités.

La fertilité après les premières chaleurs a été également calculée pour ces 3 périodes.

la fertilité globale est donnée pour une période de lutte identique à celle des brebis traitées. Less gestations établies à la suite d'une saillie lors d'un retour en oestrus sont ainsi prises en considéra. tion. 


\section{RÉSULTATS}

I. Déclenchement de l'cestrus pendant l'ancestrus post-partum

a) Influence du moment de l'année et de PMSG sur le déclenchement de l'astrus après la mise bas (tabl. I).

Race Ile-de-France.

Pendant la saison sexuelle, les pourcentages de brebis en oestrus sont respectivement de 87,3 et 79,5, pour celles recevant le progestagène seul et celles recevant en outre une injection de PMSG; la différence n'est pas significative.

Par contre, ces deux pourcentages sont significativement supérieurs à ceux des animaux témoins, moins de 42 à $5 \mathrm{I}$ jours après mise bas.

Les pourcentages de retours en astrus chez les brebis non gestantes sont identiques chez les animaux traités et chez les animaux témoins.

Le traitement permet en outre, une synchronisation des chaleurs (fig. I).

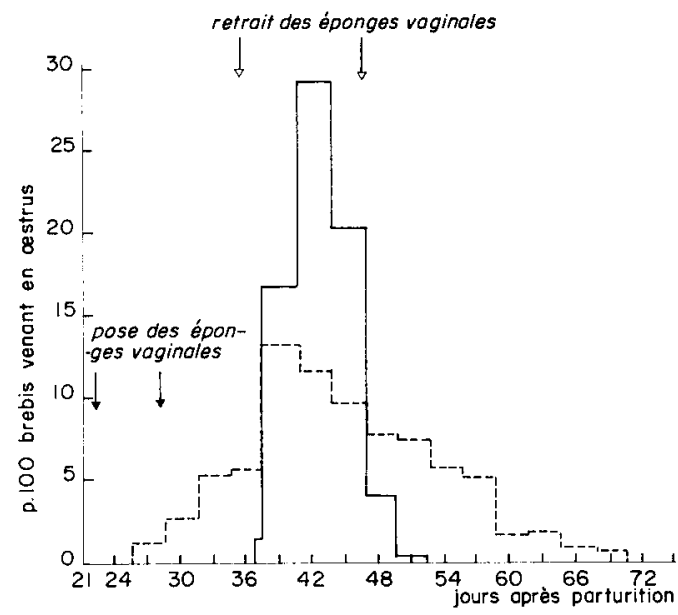

IiIg. 1. - Apparition de l'aslrus après parlurilion

Brebis, race Ile-de-France. Automne

L.es pourcentages sont exprimés par rapport au nombre de brebis mises à la lutte

- - brebis traitées avec le progestagène

- - brebis témoins

- Pendant l'ancestrus saisonnier deux périodes doivent être considérées : l'une correspondant à la période d'anœstrus complet (mars-avril), l'autre s'étalant sur les mois de mai et juin.

- Pendant les mois de mars-avril seulement I2,8 p. Ioo des animaux traités avec le progestagène viennent en cestrus. PMSG augmente le pourcentage de brebis en oestrus à 88 , I, valeur identique à celle observée pendant la saison sexuelle. En mars-avril, même avec PMSG, les animaux ne peuvent être rendus cycliques puisque le pourcentage de retours en ostrus est de $2, \mathrm{I}$. 
PROGESTOGÈNE ET FERTILITÉ PENDANT L'ANGE'TRUS

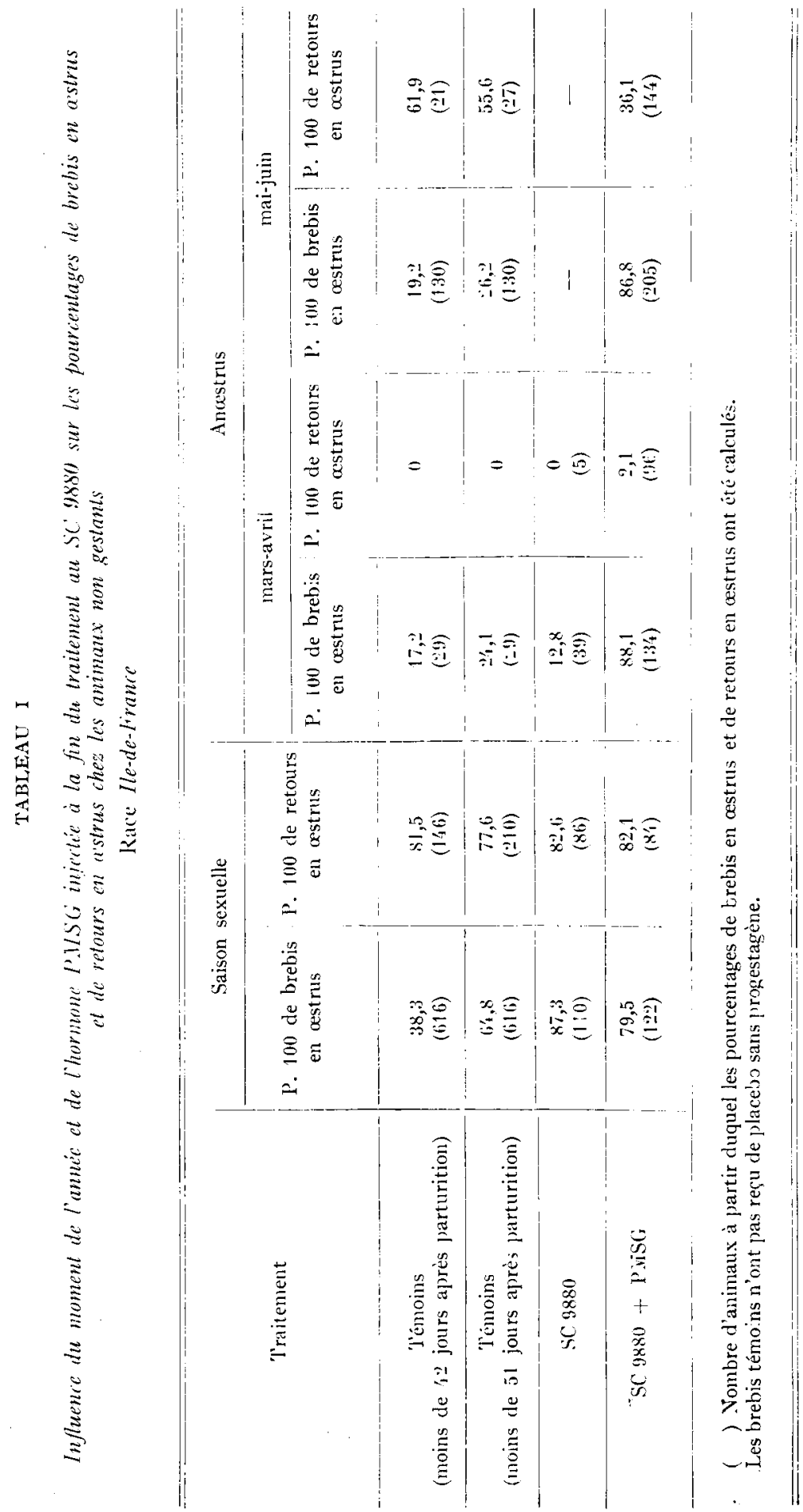

Armales de Zootechnie. - ig58. 
- Pendant les mois de mai et juin, 1'utilisation systématique de PMSG n'a pas permis de juger de l'efficacité du progestagène seul pour induire l'œestrus. PIISG associée au progestagène conduit à 86,8 p. Ioo de brebis en oestrus. Pendant cette période, 36, I p. Ioo des animaux non gestants ont présenté des retours en chaleurs, c'est-à-dire, une reprise partielle d'activité cyclique. Ce pourcentage est cependant nettement inférieur à celui de la saison sexuelle.

Race Préalpes (tabl. 2).

- Pendant la saison sexuelle, 95,8 p. Ioo des brebis viennent en cestrus à la suite du traitement à l'aicie de progestagène et PMSG.

- Pendant les mois d'avril-mai, 97,5 p. Ioo des brebis traitées recevant une supplémentation PMSG ont des chaleurs. Le pourcentage de retours en ostrus des brebis non fécondées est de 23,6 p. I00. Peu de témoins viennent en oestrus moins de 5 I jours après parturition.

\section{TABLEAU 2}

Pourcentages de brebis en oestrus et de retours en ostrus chez les brebis non gestantes après traitement associant SC 9880 et P.MSG

Race Préalpes, en période d'anoestrus (avril-mai)

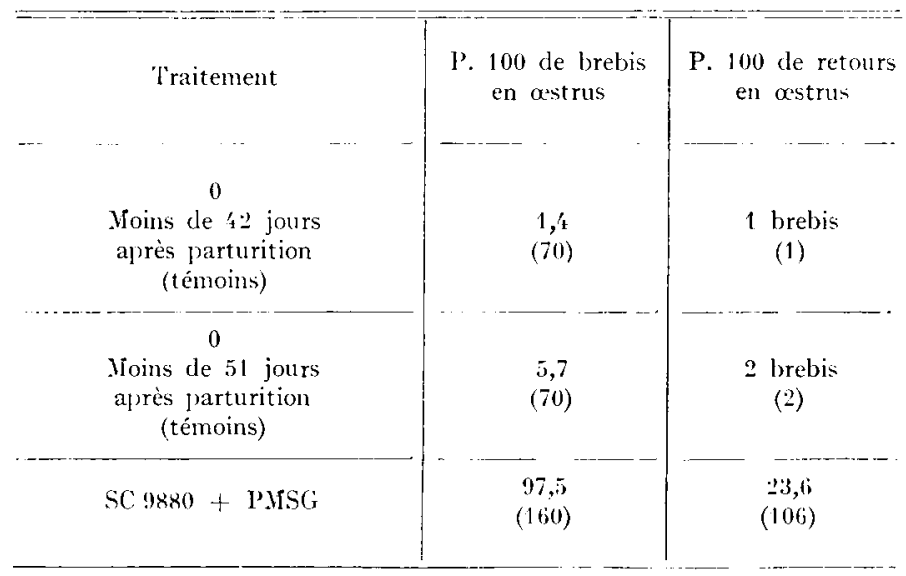

( ) Nombre d'animaux à partir duquel les pourcentages de ventes et de retours en ostrus ont été calculés.

Les brebis témoins n'ont pas reçu de placebo sans progestagìne.

b) Infuence des astrogènes sur le déclencinement de l'cestrus (tabl. 3).

Les œstrogènes injectés à la fin du traitement progestatif, en période d'anœstrus. augmentent le pourcentage d'animaux en cestrus. Cette augmentation est hautement significative. Mais les retours en œstrus pendant cette période ne sont pas accrus pour autant.

Injectés au début des chaleurs, ils augmentent la durée de celles-ci (fig. 2). 


\section{TABLEAU 3}

Influence des ostrogènes injectés à la fin du traitement au SC 9880 sur les pourcentages de brebis en cestrus et de retours en astrus chez les animaux non gestants Race Ile-de-France, en période d'anœstrus

\begin{tabular}{c|c|c}
\hline Traitement & $\begin{array}{c}\text { P. 100 de brebis } \\
\text { en oestrus }\end{array}$ & $\begin{array}{c}\text { P. 100 de retours } \\
\text { en oestrus }\end{array}$ \\
\hline Pas d'ostrogènes & $\begin{array}{c}74,2 \\
(287)\end{array}$ & 29,9 \\
$(174)$
\end{tabular}

**Différence hautement significative $\mathrm{P}<0,01$.

( ) Nombre d'animaux à partir duquel ont été calculés les pourcentage: de brebis en oestrus et de retours en cestrus.

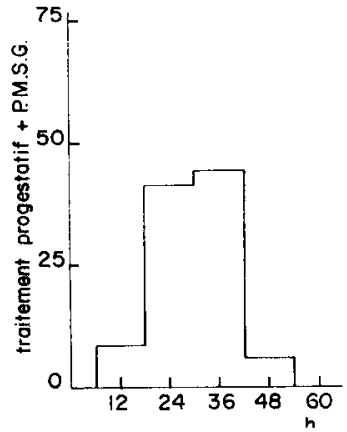

Traitement progestatif + PMSG

$(2 a)$

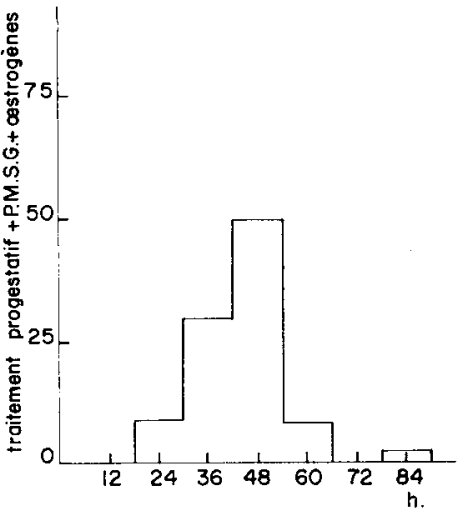

Traitement progestatif + PMSG + cstrogènes $\left(\begin{array}{ll}2 & b\end{array}\right)$

líg. 2. -- Infuence des astrogènes injectés au début des chaleurs sur la durée de l'astrus Brebis race Préalpes. Printemps 
c) Infuence de l'allaitement (tab1. 4).

Que les brebis soient taries ou non, le traitement progestatif associé ou non avec PMSG déclenche 1'œstrus chez 82 à 92 p. Ioo des animaux.

\section{TABIEAAU 4}

Influence de l'allaitement sur l'apparition de l'cstrus après traitement au SC 9880 Pourcentages de brebis en ostrus et de retours en ostrus chez les animaux non gestants

Race Ile-de-France

\begin{tabular}{|c|c|c|c|c|}
\hline \multirow[b]{2}{*}{ Lot expérimental } & \multicolumn{2}{|c|}{ Saison sexuelle } & \multicolumn{2}{|c|}{ Anoestrus } \\
\hline & $\begin{array}{l}\text { P. } 100 \text { de brebis } \\
\text { en oestrus }\end{array}$ & $\begin{array}{c}\text { P. } 100 \text { de retours } \\
\text { en œstrus }\end{array}$ & $\begin{array}{l}\text { P. } 100 \text { de brebis } \\
\text { en ostrus }\end{array}$ & $\begin{array}{l}\text { P. } 100 \text { de retours } \\
\text { en ostrus }\end{array}$ \\
\hline Brebis taries & $\begin{array}{r}85,0 \\
(127)\end{array}$ & $\begin{array}{l}85,4 \\
(96)\end{array}$ & $\begin{array}{r}92,9 \\
(141)\end{array}$ & $\begin{array}{l}51,3 \\
(115)\end{array}$ \\
\hline Brebis allaitantes & $\begin{array}{l}81,7 \\
\left(10^{\prime}\right)\end{array}$ & $\begin{array}{l}77,3 \\
(75)\end{array}$ & $\begin{array}{l}86,9 \\
(122)\end{array}$ & $\begin{array}{l}29,9 * * \\
(97)\end{array}$ \\
\hline
\end{tabular}

** Différence hautement significative $\mathrm{P}<0,01$.

( ) Nombre d'animaux à partir duquel les pourcentages de brebis en œstrus et de retours en œstrus ont été calculés.

Si le pourcentage de retours en chaleurs chez les brebis taries n'est que très peu supérieur à celui des brebis allaitantes, pendant la saison sexuelle $(85,4$ et 77,3 respectivement), la différence entre les pourcentages de retours en œstrus de ces 2 catégories d'animaux est hautement significative pendant la période d'anœstrus $(5 \mathrm{I}, 3$ et 29,9$)$.

d) Autres facteurs.

La dose de progestagène, l'intervalle mise bas-début du traitement, l'injection de PMSG 12 jours avant le tr xite nont prozestatif, la durée de ce traitement, le moment d'injection de PMSG, sont s ans effet sur la fréquence, le pourcentage et le délai d'apparition de l'œstrus.

e) Existonce des ovulations silencieuses après traitenent progestatif.

On peut supposer l'existence d'ovulations silencieuses après traitement progestatif. En effet, particulièrement en mai-juin et pendant la saison sexuelle, les brebis ne venant pas en ostrus dans les 5 jours qui suivent le retrait des éponges vaginales, et l'injection de PMSG ont parfois des chaleurs I 8 à 2 I jours aptès la fin du traitement. Ces œstrus devraient s'ajouter aux retours donnés dans les tableaux I et 2 . Nous n'en avons pas tenu compte dans cette première partie, mais ils seront pris en considération lors de 1 a présentation des résultats de fertilité. 


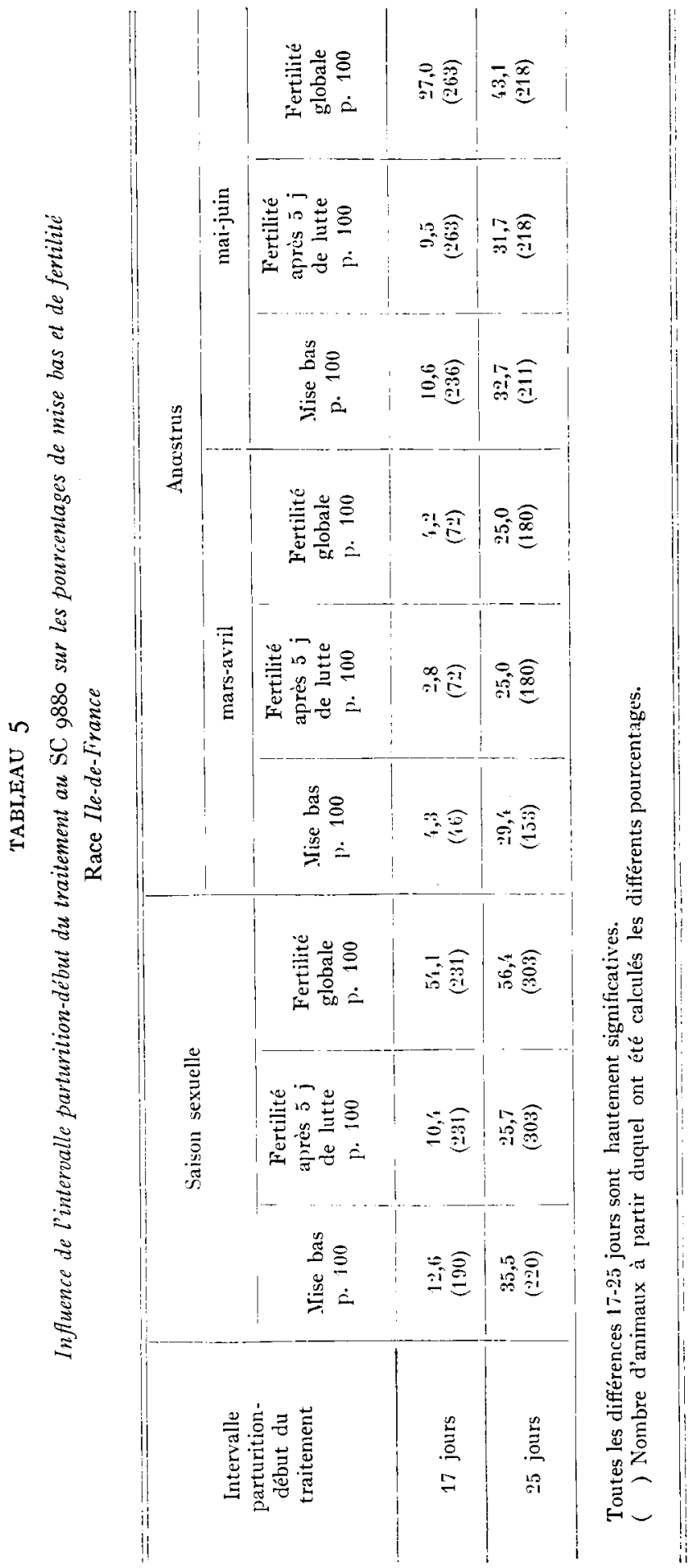




\section{Fertilité}

Les pourcentages de mise bas et de fertilité après 5 jours de lutte chez les brebis traitées sont faibles, mais ils permettent de dégager plusieurs influences.

a) Infuence de l'intervalle parturition-début du traitement progestatif (tabl. 5 et 6).

Pour les brebis de race Ile-de-France, le pourcentage de mise bas est de I2,6, pendant la saison sexuelle, lorsque l'intervalle n'est que de I7 jours. Il est égal à 35,5 lorsque l'intervalle est de 25 jours.

\section{TABLEAU 6}

Pourcentages de mise bas et de fertilité des brebis témoins pour différentes périodes après parturition et influence de l'intervalle parturition-début du traitement au SC 9880 sur les pourcentages de mise bas et de fertilité chez les brebis traities

Race Préalpes, en période d'anostrus (avril-mai)

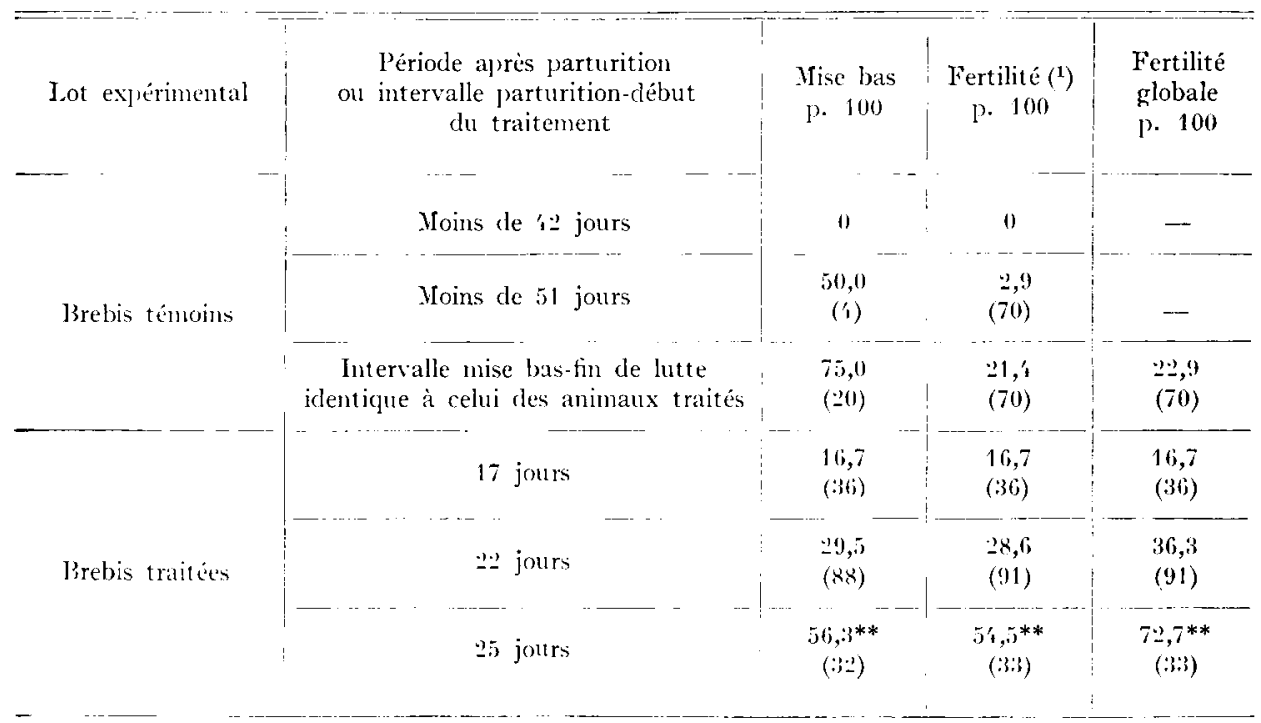

(1) Fertilité après aj jours cle lutte pour les brebis traitées.

Fertilité pour la période considérée pour les animaux témoins.

** Différences 17-:25 jours hautement significatives $\mathrm{P}<0,01$.

Les différences $: 2-2-25$ jours sont toutes significatives $\mathrm{P}<0,05$.

L'augmentation du pourcentage de mise bas est aussi importante en mars-avril et en mai-juin, lorsque l'intervalle passe de $I 7$ à 25 jours.

Pour les brebis de race Préalpes, l'accroissement de l'intervalle parturition-début du traitement progestatif a un effet semblable sur le pourcentage de mise bas. Ainsi, en période d'anœestrus, ces pourcentages sont respectivement de $16,7,29,5$ et 56,3 pour des intervalles de $I 7,22$ et 25 jours. 
L'augmentation de l'intervalle parturition-début du traitement accroît donc de façon significative, le pourcentage de mise bas, quels que soient la race et le moment de l'année. Il en est de même pour la fertilité après 5 jours de lutte.

b) Infuence de la race (tabl. 5 et 6 ).

En période d'anœstrus, la comparaison est très nettement en faveur des animaux de la race Préalpes, particulièrement, lorsque l'intervalle est de 25 jours : pourcentages de mise bas respectivement de 32,7 et 56,6 pour les brebis de race Ile-de-France (mai-juin) et pour les brebis de race Préalpes (avri1-mai).

Pour un intervalle de mise bas-début du traitement variant entre I 7 et 25 jours, chez les brebis de race Préalpes, en saison sexuelle, le pourcentage de mise bas est de 30,7 et celui de la fertilité après 5 jours de lutte de 29,2 p. Ioo. Ces résultats sont également supérieurs à ceux obtenus chez des animaux de race Ile-de-France dont le traitement a débuté $\mathrm{I} 7$ jours après parturition et comparables à ceux obtenus lorsque le traitement débute 25 jours après mise bas.

c) Influence de la date de traitement (tabl. 5).

Chez les brebis traitées de race Ile-de-France, les pourcentages de mise bas et de fertilité après 5 jours de lutte ne sont que très peu influencés par la saison. Mais celle-ci a un effet considérable sur la fertilité globale. Elle est beaucoup plus élevée pendant la saison sexuelle qu'aux autres périodes de l'année. Ceci résulte du fait que, pendant la saison de reproduction, le pourcentage de retours en ostrus chez les animaux non gestants est plus important que pendant la période d'anœestrus. Lors de ces retours le pourcentage de mise bas est normal.

d) Infuence de l'allaitement (tab1. 7).

Chez les brebis Ile-de-France faisant l'objet de la comparaison, l'intervalle parturition-début du traitement est de I7 jours, le pourcentage de mise bas est donc

TABLEAU 7

Influence de l'allaitement sur les pourcentages de mise bas et de fertilite?

(Brebis Ile-de-Irance)

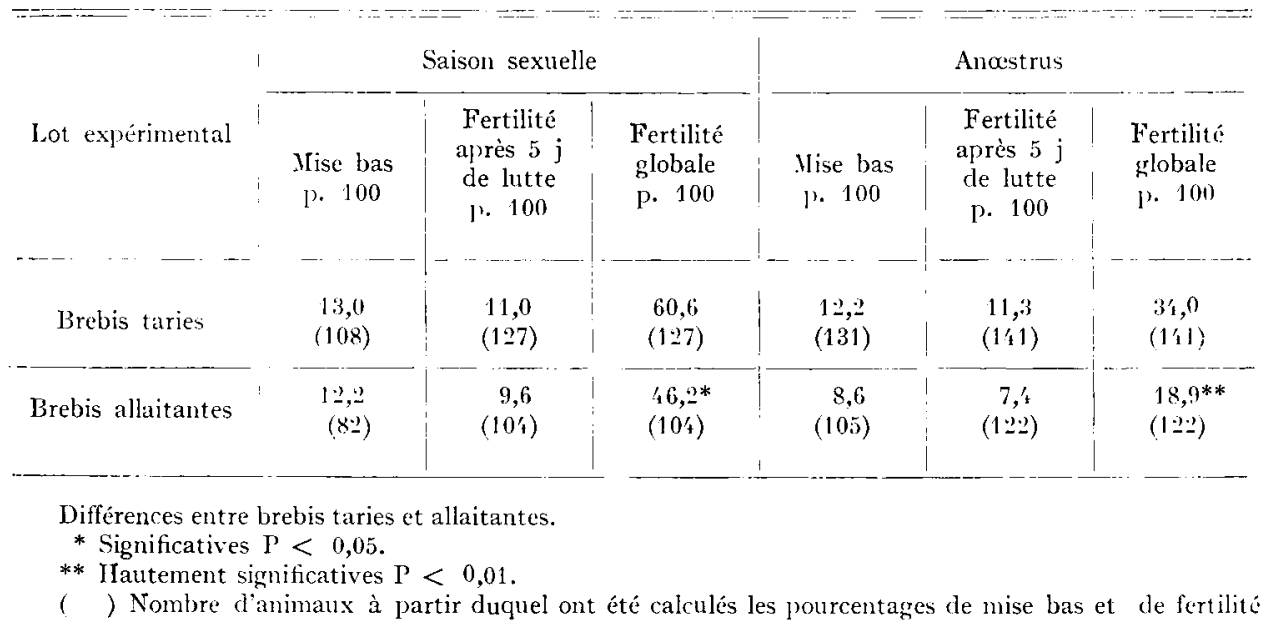




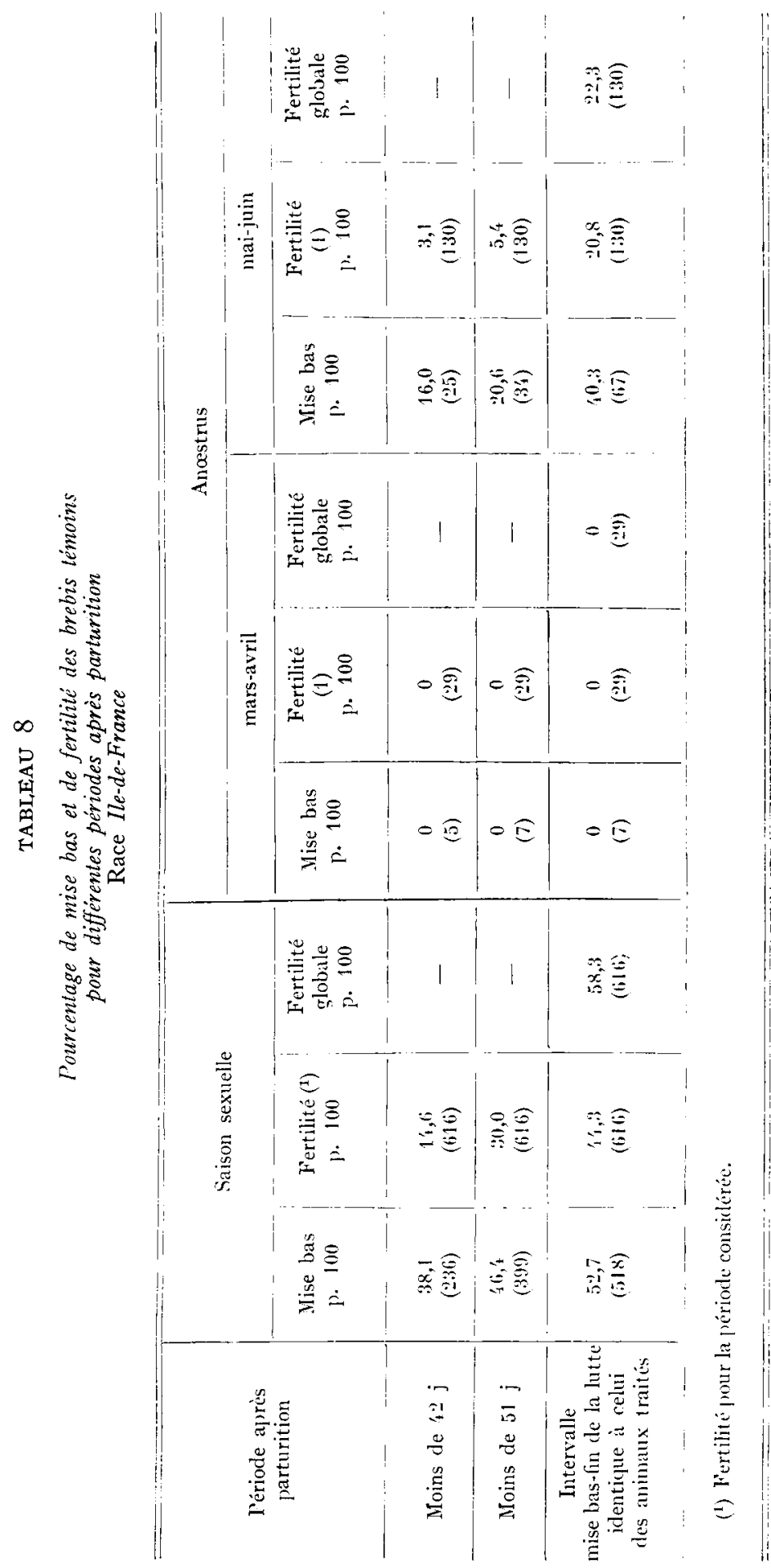


taible (environ I2 p. IOo). Mais la fertilité globale est plus élevée chez les̀ brebis taries, les retours en cestrus étant plus nombreux que chez les brebis allaitantes. Cet effet est très marqué pendant la pério le d'anostrus.

e) Pourcentage de mise bas chez les animaux témoins (tabl. 5 et 8).

En saison sexuelle, le pourcentage de mise bas augmente au fur et à mesure que l'on s'éloigne de la parturition. Bien que le pourcentage de mise bas soit faible, moins de 42 jours après 1 mise bas, il est cependant plus élevé que celui obtenu chez les animaux traités avec un traitement débutant 17 jours après parturition : 38 , I p. Ioo et I2,6 p. Ioo respectivement pour les brebis témoins et les brebis traitées $(\mathrm{P}<0,0 \mathrm{I})$.

I a comparaison du pourcentage de mise bas moins de $5 \mathrm{I}$ jours après parturition chez les animaux témoins, (46,4 p. IOO) avec celui des animaux dont le traitement a débuté 25 jours après mise bas $(35,5$ p. I00), montre encore une différence en faveur des animaux témoins, mais $1 x$ différence est moins importante. Elle est cependant significative $(\mathrm{P}<0,05)$.

En mars-avril, le pourcentage de mise bas est nul quel que soit l'intervalle après parturition. En mii-juin, ce pourcentage est faible, mais il augmente également au fur et à mes̀ure que l'on s'éloigne de 1 a mise bas. La fertilité globale reste faible $(22,3 \mathrm{p}$. IOO) et est très nettement inférieure à celle des animaux traités $(\mathrm{P}<0,0 \mathrm{I})$.

\section{DISCUSSION}

\section{a) Obtention de l'astrus}

Il est aisé de déclencher l'oestrus très rapidement après la mise bas par un traitement progestatif associant ou non, suivant la saison, 1'hormone PMSG.

Le progestagène associé avec PMSG permet, comme l'association progestéronePMSG, d'obtenir l'œstrus chez un nombre élevé de brebis pendant la période de repos sexuel alors que PMSG, seule, provoque l'ovulation mais est peu efficace pour déclencher l'œestrus et que la progestérone seule ne permet pas, dans la plupart des cas, d'obtenir œstrus et ovulation (RAESIDE et LAMOND, I956; MCDONAI,D, I96I).

Le progestagène seul suffit en saison sexuelle. Une supplémentation avec PMSG ne se révèle indispensable que pour déclencher l'œestrus en période d'anœestrus. La faible teneur en hormones gonadotropes pendant la période d'anostrus (THIMONIER et Mauléon, I967, I968) nécessite l'intervention d'un tel facteur.

L,e rôle des cestrozènes agissant sur le système nerveux central sensibilisé par les progestagènes est conforme aux observations de Robrssox (I959).

\section{b) Obtention de la gestation}

L'obtention de la gestation est beaucoup plus difficile. Le pourcentage de mise bas lors de l'œestrus induit est faible. Esst-ce dî̀ à un intervalle parturition-première saillie trop court, ou est-ce lié au problème de l'utilisation des progestagènes ?

Bien que les travaux sur la synchronisation de l'œstrus à l'aide des progestagènes fassent apparaître une diminution de la fertilité (FoORD, I966; RoBERTs, I 966 ; Robinson et Lamond, Ig66; Wishart, ig66-Ig67 ; Cognié et Colas, ig68), celle-ci 
n'est pas aussi brutale que celle résultant d'un intervalle parturition-saillie court. Cette diminution peut même être nulle lorsque le traitement avec le progestagène débute peu de temps après l'ovulation (tabl. 9) ou est effectué chez des agnelles ('THIMONiER et al., rg68).

\section{TABIEAU 9}

Infuence du moment du début du traitement progestatif au cours du cycle sur le pourcentage de mise bas

Traitement progestagène : $45 \mathrm{mg}$ SC 9880 . Durée 14 jours Race Ile-de-France. Insémination artificielle

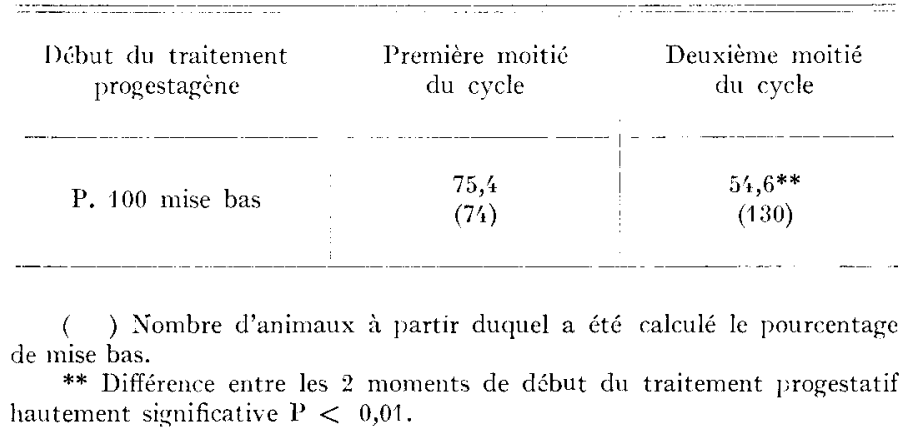

Il semble donc que cette diminution de fertilité soit propre à la situation physiolosique des brebis peu de temps après la mise bas.

Cependant, 3 causes au moins peuvent encore réduire la fertilité : influence résiduelle du progestagène sur l'utérus, qualité des ovules et inaptitude du tractus à assurer le développement de 1'œuf fécondé.

L'augmentation de l'intervalle parturition-début du traitement ou, ce qui ne peut être dissocié dans nos différentes expérimentations, 1'augmentation de 1'intervalle parturition-saillie, entraîne une augmentation du pourcentage de mise bas. Cette amélioration est peut-être liée uniquement à l'état utérin qui empêche l'implantation trop tôt après la parturition (GORDON, I958). Il faut attendre une certaine remise en état de l'utérus avant de pouvoir provoquer l'installation d'une nouvelle gestation (HEAP, ALLEN et LAMMING, I963).

L'inefficacité de l'injection de PMSG, 8 jours après la mise bas tendrait à montrer que le rétablissement de l'utérus n'est pas suffisant ou que l'injection de PMSG n'est pas faite au moment le plus favorable par rapport au début du traitement avec le progestagène.

Ce rôle probable de l'utérus est mis en évidence également chez les animaux témoins; le pourcentage de mise bas augmente au fur et à mesure que l'on s'éloigne de la parturition.

Cette augmentation du pourcentage de mise bas a déjà été notée par d'autres auteurs (WAGNER, I964; HULET et FOOTE, I967).

Les différences raciales peuvent être expliquées si l'on admet un rôle des ovulations silencieuses dans le rétablissement d'un état utérin normal. Les ovulations 
silencieuses se produisent plus fréquemment chez les brebis Préalpes que chez les brebis Ile-de-France, aussi bien pendant l'ancestrus saisonnier (ThImonier et MaUI,ÉON, I968), que pendant l'ancestrus de lactation (Maulíon, non publié).

Le pourcentage de mise bas lors des retours en ostrus chez les animaux non gestants après une première saillie, est normal. Aussi, lorsque les retours en oestrus existent, la fertilité globale augmente. Ia saison et l'allaitement des jeunes influent sur l'apparition de ces retours en œstrus, donc sur la fertilité globale.

Rendre les brebis cycliques pendant la période de repos sexuel permettrait d'augmenter considérablement la fertilité globale. On peut donc se demander avec HULET et FOOTE ( $1967 b$ ), si les manipulations photopériodiques associées au traitement progestatif ne permettront pas de résoudre le problème de l'ancestrus, en attendant que soit trouvée la solution à la faible fertilité lors de l'œstrus induit.

Rę̧u pour publication en mai 1968.

\title{
REMERCIEMENTS
}

Nous adressons nos remerciements à la Fédération nationale ovine et aux éleveurs qui ont participé aux expérimentations ainsi qu'à la Société Clin-Byla qui a fourni l'acétate de fluorogestone nécessaire aux différents essais.

\section{SUMMARY}

\author{
INDUCTION OF EESTRUS AND PREGNANCY IN THE EWE \\ DIRING "POST PARTUM" ANESTRUS BY MEANS OF PESSARIES \\ IMPREGNATED WITI FLUOROGESTONE ACETATE
}

'The factors influencing hormonal induction of cestrus and pregnancy during post-partum anoestrus in the ewe were studied at various times of the year.

Irrespective of the season, astrous induction by intravaginal progestagen treatment 32 to 35 days after lambing was experienced in 80 to 98 per cent of the ewes. However, a PMSG supplementation was required.

The recurrence of aestrus in the non-pregnant animal was higher in ewes dry within 2 to 4 days after lambing, than in lactating ewes, particularly during the seasonal anostrous period.

Fertility after the induced cestrus was very low. It increased with the time interval between lambing and the begimning of treatment. In Ile-de-France ewes, the fertility rate increased from io to 35 per cent as the time interval increased from 17 to 25 days.

There were breed differences. For a given time interval after lambing, within the ancestrous period, the fertility rates after cestrous induction were 32 and 55 per cent, for Ile-de-France and Prialpes respectively. The recurrence of cestrus was more frequent in dry ewes than in lactating ewes. It is therefore possible to improve the total breeding efficiency.

Within the breeding season, fertility was the same in the control as in the progestagen-treated ewes. However, the progestagen treatment allowed synchronization of heat.

In both control and treated ewes, fertility after the first cestrus increased depending upon the time elapsed since parturition. 


\section{RÉFÉRENCES BIBLIOGRAPHIQUES}

Allev D. M., LAmMing G. E., 1960. The induction of breeding activity in lartating ewes duringr anostuus. J. Reprod. Ferl., 1, 21 3-222.

Convie Y., Colas G., rg68. Évolution des techniques cle reprcduction rhez les Ovins : synchronisation des chaleurs, obtention de deux agnelagres par an, mise précoce à la lutte des agnelles, insémination artificielle. Pâtre, $153,17-24$.

Cole H. H., Mrller R. F., r933. Artificial induction of ovulation and ostrum in the ewe during anœtrum. Amer. J. Physiol., io ${ }^{-} .53$.

DAvzier L., Wixtexberger S., i952, Dáclenchement simultané de l'astrus dans un lot de brebis avec possibilité de gestation ultérieure. Inn. Zootech., 4, 49-52.

Desvignes A., D.arpoux R., i 964 . Valeur d'élevage des brebis utilisées pour le croisement industriel avec différentes races de béliers. Bull. Tech. Inf. Ingrs Serv, Igric., 195, 893-90:.

Durs R. H., 1952. Induction of cestrus and ovulation in anestrual ewes by use of progesterone and pregnant mare serum. J. Anim. Sci., 11, 792.

Foord H. E., I 965 . Observations on the use of progesteron: inpregnated tampons in a herd of $\mathrm{Dorsel}$ Hill sheep. Vel. Rec., 73, 461 .

GoRDon I., I958. The use of progesterone and serum gonadotrophin (PMS) in the control of fertility in sheep. II Studies in the extraseasonal production of lambs. J. Igric. Sci., 50, $152-197$.

Gorbon 1., I $963 a$. The induction of pregnancy in the andstrous ewe by hormonal therapy. 11. Progesterone-pregnant mare's serum applications in ancestrus. $/$. . Igric. Sci, 60, 43-66.

Gordon I., $1963 b$. The induction of premnancy in the ancestrous ewe by hormonal therapy. III. The use of repeated progesterone-pregnant mare's serum therapy. I. Lgric. Sci., 60, 67-85.

Granger W.. ig47. Conception in lactating ewes. flosl. Vol. J., 23, $14,3-145$.

IIAFE E. S. E., 1952. Studies on the breeding season and reproduction of the ewe. I. Igric. Sci., 42, $189-265$.

IIANSEL W., 1964. Livaluation of methods for controlling the estrous cycle. Proc. Coni. on estrous cycle control in domestic animals: July 9. 10. 1964. Jincoln, Nobraska, Misc. Pub., 1-16.

IIEAP R. B., AlLen D. M., LAMmis; G. E., I 963 . Influence of the induction of breeding activity in anestrous sheep on the levels of some rhemical constituents of $u^{+}$erine washings. J. Reprod. Fert., 5, 209-2I 5 .

IIUlet C. V., Foo'te W. C., 1976 a. Induction of fertile estrus in lactating and dry ancestrous ewes using . oral progestagene and repeated PIS treatment. J. Amim. Sci., 26, 545-548.

Hilet C. Y. Foote W. C., ig $67 b$. Physiological fartors afferting frequency and rate of lambing. $J$. Anim. Sci., 26, 553-562.

IfE; J. L., 1964. Inhibitory effect of lactation on the breding activity of the ewe. Vatur, 233, $1089^{-1090 .}$

MCDonald M. F., 196. Studies of the resonse of the ancestrous ewe treated with promesterome and pregnant mare serum. J. Agric. Sci, 58, 3)7-405.

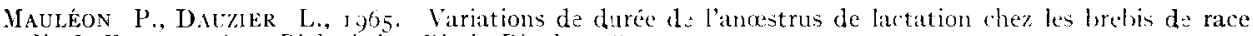
lle-de-France. Inn. Biol. Anim. Bioch. Biopltys., 5, $1.3-1+3$.

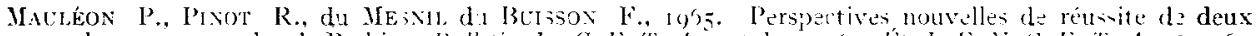

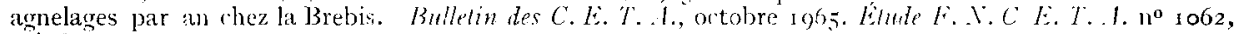
3 à 8.

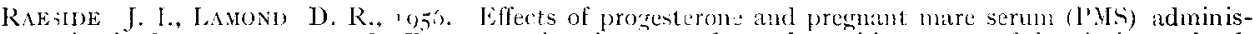
tration in the anostrous ewe. I. (Eitrus, ovarian changes and gonadotrophic potency of the pituitary gland. lust. J. Agric. Res., 7, 50 - joo.

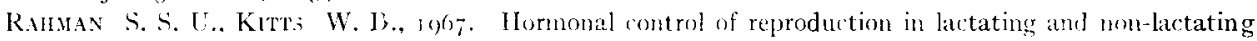
ancstrous ewes. Can. J. Inim. Sci, 47,65.

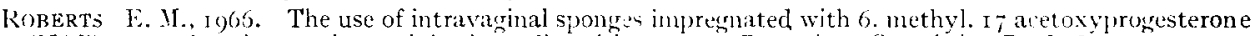
(MAP) to synchronize ovarian activity in cyclic mérino ewes. Proc. Aust. Soc. Anim. Prod., 6, 32-37.

Risinson T. J., I954. Fertility of ancestrous ewes following injection of progresterone and presnant mare serum (PMS). Altsir. J. Agric. Res., 5, 730-736.

RoBINion T. J., I9.5\%. The estrous cycle of the ewe and doe in CoLE H. H., Cupps P. T. Reproluction in Domestic Aimmals, Vol r, 29i-333, Acarl. Press, New York and London.

Robivsox T. J., i 965 . Lise of progestagene impregnated sponges inserted intravaginally or subcutaneously for the control of the ostrous cycle in the sheep. Valwe' 206, 39-4I.

Robinson T. J., I.Amond D. R., 1066. Control of reproduction in sheep and cattle. Proc. Aust. Soc. Anim. Prod., 6, Iо-I8.

Thimonier J., Mauléon P., 1967. Variations saisonières des activités hypophysaires des brebis de race lle-de-France. Coll. Int. "Pholorégulation de la reproduclion che: les oiseaux el les Mammifives "Nontpellier i 7 -22 juillet 1967 (sous presse). 
Fumonier J.. Maliken P'., jo68. Variations saisomieres du comportement d'cestrus et des activités ovarieune et hypophysaire chez les Ovins. Ann. Biol. Anim. Bioch. Biophys. (sous presse).

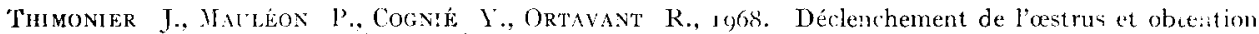
précoce de gestations che\% les agnelles à l'aide d'éponges vaginales imprégnées de l'acétate de fiuorogestone. Anm. Yowtech., 17, $275^{-288}$.

Wagner J.F., rofit. Hormonal control of reproductive activity in the ewe. Proc. Conf. on esiruns crele control in domeslic annimale: July 9.'0. 1964. I.incoln, Nebraska, Iisc. Pub. 28-44.

Wisiart I). l:. lo66. The indurtion of earlier breeding activity in sheep. A comparison between the use of vasectomised rams and the use of intravaginal pessaries impregnated with a new progestin. Vet. Rec., 79, 356-3.58.

WismarT D. F., lus. Symehronization of estrus in sheep: the use of pessaries. Vet. Rec, 81. $276-287$.

Williams S. II., (iarricus I. S., Norton H. W., Nalbanion A. V., I956. 'The occurence of estrus in pregnant ewes. J. Anim. Sci., 15, $97^{8-983}$.

7.Avadosky B.Y., 1945. Direction du processus de reproduction des animax. (en Russe). Ed. OG I 2. 